\title{
Hypertension as an Unusual Cause of Proliferative Retinopathy: Case Report and Literature Review
}

\author{
Rami Hasan Saleem Abu Sbeit ${ }^{a}$ Osman Abdelzaher Mohammed ${ }^{a}$ \\ Laith Ishaq Alamlih ${ }^{b}$ \\ aDepartment of Ophthalmology, Hamad Medical Corporation, Doha, Qatar; ${ }^{b}$ Division of \\ Rheumatology, Department of Medicine, Hamad Medical Corporation, Doha, Qatar
}

\section{Keywords}

Hypertensive retinopathy $\cdot$ Malignant hypertension $\cdot$ Proliferative retinopathy $\cdot$ Retinal neovascularization - Vitreous hemorrhage

\begin{abstract}
Malignant hypertensive retinopathy is associated with characteristic fundus findings that typically do not include proliferative retinal vascular changes. We present the case of a 34-yearold patient who had bilateral decreased vision and was found to have malignant hypertension with hypertensive retinopathy changes along with unforeseen bilateral neovascularization and vitreous hemorrhage. Detailed history and extensive systemic and ophthalmic workup failed to reveal an alternative explanation for her proliferative retinopathy. Blood pressure control and panretinal photocoagulation halted further deterioration. Malignant hypertensive retinopathy can rarely cause profound retinal ischemia leading to retinal neovascularization. This case further supports the presence of "proliferative hypertensive retinopathy" that needs to be identified and addressed urgently through collaboration between internists and ophthalmologists.
\end{abstract}

\section{Introduction}

Hypertension is a risk factor for several ocular vascular diseases and can directly cause retinopathy, choroidopathy, and optic neuropathy. Many fundus findings are associated with hypertensive retinopathy such as arteriolar narrowing, arteriovenous nicking, cotton wool spots, intraretinal hemorrhages, exudates, and disc swelling. However, few reports support

\section{Karger $\stackrel{\text { ! }}{=}$}


the notion that in extreme cases of hypertension, severe retinal ischemia can provoke retinal neovascularization. Nonetheless, the existence of "proliferative hypertensive retinopathy" has been a controversy since it was proposed in 2016 [1]. We hereby present a case of malignant hypertensive retinopathy who developed proliferative changes in the absence of other ocular or systemic causes. We performed an extensive literature review exploring such an association.

\section{Case Presentation}

Thirty-four-year-old Asian lady presented to the emergency department with blurred vision and continuous headache of 1-week duration. She denied any history of ocular pain, redness, photophobia, or trauma. The review of systems was unremarkable including for symptoms suggestive of autoimmune diseases such as malar rash, photosensitivity, mouth ulcers, joint pain, Raynaud's, or sicca. She was diagnosed few years earlier with hypertension but was not compliant with her medications or follow-up. There was no history of smoking, alcohol intake, or drug abuse. Her family history was noncontributory. In the emergency room, her vital signs were normal except for remarkably high blood pressure (BP) of 290/160 $\mathrm{mm}$ Hg. She had equal BP and pulses bilateral. Cardiovascular, respiratory, and abdominal examinations were unremarkable. Her best-corrected visual acuity (VA) was 6/60 in the right eye (OD) and 3/60 in the left eye (OS). Refraction showed mild compound myopic astigmatism. Intraocular pressures were within normal limits. Anterior segment examination was unremarkable and dilated fundus examination revealed bilateral optic disc swelling, posterior pole extensive lipid exudation, narrowing of arterioles, mild venous tortuosity, vascular loops and anastomosis, intraretinal hemorrhages, and cotton wool spots consistent with malignant hypertensive retinopathy as per Wong and Mitchell classification. The OD showed mild vitreous hemorrhage ( $\mathrm{VH}$ ) and an underlying neovascularization elsewhere (NVE), while the OS did not show clinically evident retinal neovascularization (Fig. 1). There was no vitritis in either eye. Macular optical coherence tomography (OCT) showed bilateral neurosensory detachments and exudates, and optic disc OCT was consistent with the clinically appreciated right more than left disc swelling (Fig. 2). She was diagnosed as a case of malignant hypertension and was admitted for BP control. Her kidney and liver function tests were normal. Workup for secondary causes of hypertension including thyroid function test, urine for protein, 24-h urine metanephrines, and ultrasound doppler for renal arteries was all unremarkable. Her echo showed mildly reduced ejection fraction and severe left ventricular concentric hypertrophy suggestive of long-standing hypertension.

The patient was discharged on 3 oral antihypertensive medications and did not adhere to her appointment with ophthalmology. Nine weeks later, the patient presented with further vision deterioration and a BP of 157/78 mm Hg. Examination showed bilateral mild VH and bilateral NVE's as well as more vessel obliteration than the initial exam. At this point, her optic disc swelling had decreased and macular OCT showed persistent exudation with resolution of the neurosensory detachment. Fluorescein angiography (FA) showed bilateral severe retinal ischemia with active neovascularizations and significant macular late leakage with irregular foveal avascular zone margin (Fig. 3). Her VA was stable.

The presence of proliferative changes triggered further workup. Blood glucose measurement and HbA1c were normal; complete blood count showed normal white blood cell counts and mild anemia with normal mean corpuscular volume and hemoglobin of $11 \mathrm{~g} /$ $\mathrm{dL}$; inflammatory markers including erythrocyte sedimentation rate and C-reactive protein were normal; electrocardiogram showed no arrhythmia; echo and cardiac magnetic resonance imaging were negative for valvular vegetations; her head computed tomography was

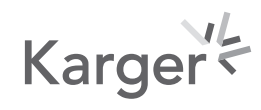



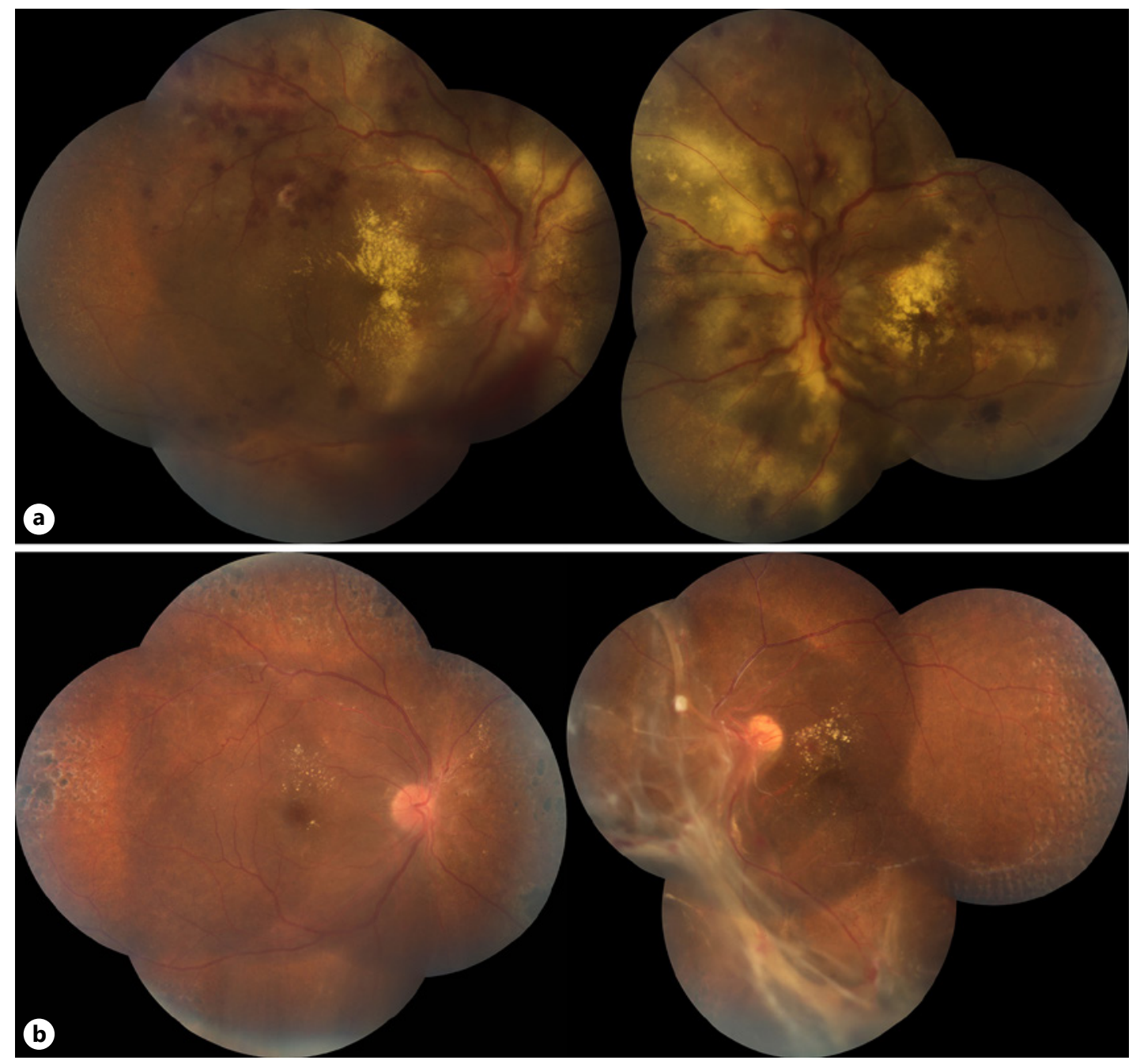

Fig. 1. a Fundus photos of both eyes on presentation showing features of malignant hypertensive retinopathy and OD VH. b Fundus photos 6 months after PRP shows regression of NVE's, pigmented laser marks and OS extrafoveal tractional membranes. OD, right eye; OS, left eye; NVE, neovascularization elsewhere; PRP, panretinal photocoagulation; $\mathrm{VH}$, vitreous hemorrhage.

unremarkable; her chest X-ray showed no hilar lymphadenopathy, and calcium level was normal; lupus anticoagulant and anticardiolipin antibody were negative. Her antinuclear antibodies came back positive. Evaluation by rheumatology excluded systemic lupus erythematosus (SLE) and other rheumatological diseases as she did not have any suggestive clinical manifestations and her laboratory workup including complement levels, double-stranded DNA antibodies, and extractable nuclear antigens panel including anti-Ro antibodies were all unremarkable. She was also found to have anti-thyroid peroxidase and anti-thyroglobulin antibodies that can have cross-reactivity with nuclear antigen and, therefore, result in a positive antinuclear antibody.

Bilateral panretinal photocoagulation (PRP) was done and she was followed closely. At her 6-month follow-up visit, her BP was well controlled, and her VA was 6/30 OD and 6/300 OS. Both eyes showed flat atrophic thinned macula with decreasing lipid exudates, regression of NVE's and pigmented PRP marks. The OD had remnant of VH and the OS showed extrafoveal tractional membranes (Fig. 1). The patient did not adhere to her appointments afterward. 


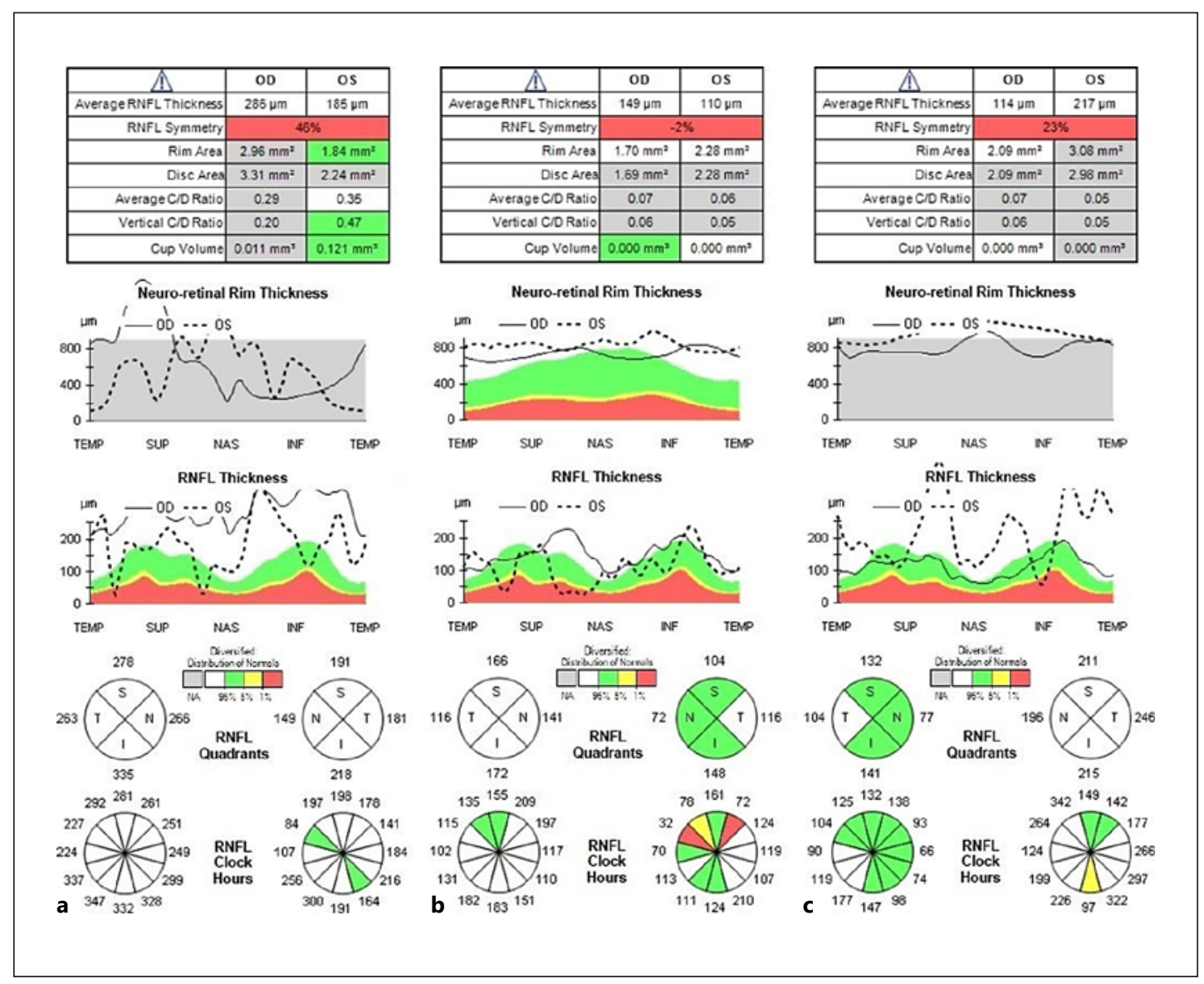

Fig. 2. Optic disc OCT on presentation (a), 9 weeks later (b), and on final follow-up (c). Note the decreasing disc edema with time. The left disc reading was falsely high in the final follow-up due to an artifact caused by the tractional membrane. OCT, optical coherence tomography.

\section{Discussion}

Retinal neovascularization attributed to hypertensive retinopathy is rarely reported in the literature. The diagnosis should be considered only after performing a thorough evaluation to exclude more common causes of proliferative retinopathy. The differential diagnosis of retinal neovascularization is vast and includes vascular disease with ischemia such as diabetes, retinal vein occlusion, ocular ischemia syndrome, hyperviscosity syndromes, and others, as well as inflammatory diseases affecting the retinal circulation as can be seen in sarcoidosis, SLE, Takayasu arteritis and others, in addition to various rare miscellaneous causes. In the current case, the presence of severe uncontrolled hypertension was the only possible explanation for such a complication. In the FA, the arm-to-retina circulation time, retinal filling time, and arteriovenous transit time were all normal. No delay in vein filling was noted. This excludes ocular ischemia syndrome and retinal venous occlusion. The absence of aneurysmal dilations of the optic nerve head and retinal arterioles at or near their major branching sites and the presence of a systemic disease explaining the findings exclude the rare entity idiopathic retinal vasculitis, aneurysms, and neuroretinitis (IRVAN). Other causes such as diabetes, hemoglobinopathies, SLE, vasculitis, sarcoidosis, familial retinopathies, embolization, and drug abuse were excluded. 

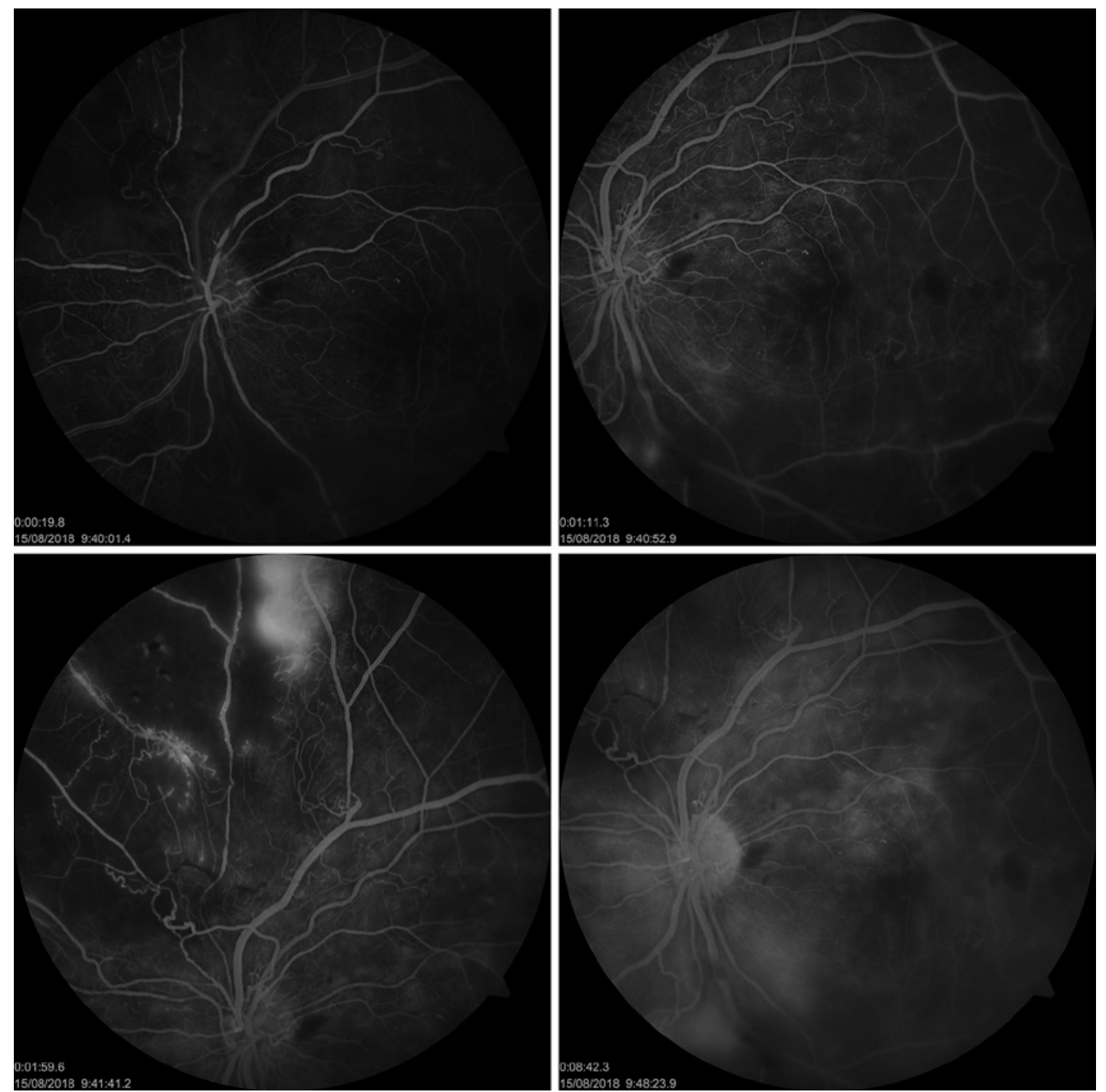

Fig. 3. FA of the OS showing normal arm-to-retina circulation time and arteriovenous transit time, severe retinal ischemia with intense dye leakage from 1 active neovascularization, widened and irregular FAZ and significant macular late dye leakage. OD showed similar but less pronounced FA findings. OD, right eye; FA, fluorescence angiography; OS, left eye; FAZ, foveal avascular zone.

The leading mechanism behind neovascularization and fibrovascular proliferation is growth factors upregulation induced by retinal ischemia. It was very recently when the term "proliferative hypertensive retinopathy" was first used to describe bilateral malignant hypertensive retinopathy with profound retinal ischemia leading to proliferative changes in a young patient [1]. Only one review article dealing with hypertensive retinopathy published in 2016 acknowledged proliferative retinopathy to be on the spectrum of hypertensive eye disease [2]. This acknowledgment was based solely on 2 case reports [1,3].

Looking at all articles listing the causes of VH in adults in the English literature, hypertension or hypertensive retinopathy were mentioned among the rare causes in only a handful. The exact causal relationship was not explored or was simply presumed in all of those articles. In fact, Lean and Gregor [4] pointed out that although systemic hypertension is frequently considered as a cause of $\mathrm{VH}$, yet in reality, it was (and still is) an unusual direct etiological factor. 
We performed an extensive literature search for reports of hypertensive retinopathy associated with retinal neovascularization or proliferative retinopathy. We included all articles in the English literature trying to explore this unique association with no restriction on the date of publishing. We found only 3 published case reports, all of which are of young patients presenting with visual complaints who were found to have very high BP readings (Table 1) $[1,3,5,6]$. All cases had features typical of hypertensive retinopathy as well as large areas of retinal ischemia and neovascularization with leakage.

The case published by Georgiadis et al. [3] had poorer vision in the OD with macular ischemia on FA and atrophy on OCT, and therefore, intravitreal anti-vascular endothelial growth factor (VEGF) was chosen over PRP to preserve peripheral vision and the eye was injected once. The OS received PRP. The neovascularization regressed in both eyes. However, 2 months posttreatment, recurrence of neovascularization OD and persistent late temporal vascular leakage OS were observed which necessitated another injection OD and augmentation of PRP OS. The patient was then followed monthly and the condition proved stable at the 12-month follow-up. Golshani et al. [5] reported a case with neovascularization of the disc and complete loss of retinal capillaries and choroidal flush. She received a single intravitreal anti-VEGF injection and 1 session of PRP in each eye. She was then lost to follow-up and showed up many months later complaining of pain due to neovascular glaucoma [6]. She was not compliant with renal dialysis or with her antihypertensive medications. These cases provide a sense that early diagnosis and intervention as well as close observation with possible augmentation of ocular treatment are all essential factors for a favorable outcome.

Literate review identified 3 other limited reports. A fundus photo of a young man with malignant hypertensive retinopathy changes along with an neovascularization of the disc was published by Abbassi et al. [7]. Another report was of a young woman with cocaine abuse who developed peripheral neovascularizations presumed to be due to ischemia from repeated episodes of hypertension and retinal vasoconstriction [8]. The third is for a patient with preeclampsia who developed peripheral neovascularization; however, with normal bilateral posterior poles, the neovascularization was attributed to low-grade disseminated intravascular coagulation rather than to hypertension per se [9]. No further details were available for these 3 cases.

The rapid persistent rise in $\mathrm{BP}$ in malignant hypertension results in arteriolar constriction that eventually leads to necrosis of the arteriolar wall smooth muscle cells decreasing their ability to constrict. This in turn results in failure of autoregulation and thus to the transmission of the high BP to the endothelium breaking down the blood-retina barrier [10], which translates to features of ischemia and leakage that are seen in hypertensive retinopathy.

It is well known that young individuals are more susceptible to hypertensive choroidopathy. This is explained by the fact that their blood vessels are more pliable and flexible than older people because of the absence of sclerosis [10]. Of note, all the patients described in the cases of hypertensive retinopathy with retinal neovascularization were relatively young. This may as well be explained by the pliable nonhyalinized retinal vessels in the young. Moreover, retinal vessel autoregulatory mechanisms can maintain vascular tone longer than choroidal vessels, and thus, the retina is expected to withstand high BP for a longer duration than the choroid before developing significant ischemia [10]. This and the wide availability of oral antihypertensive medications may explain the rarity of proliferative hypertensive retinopathy.

Controlling BP remains the most important step in the management of hypertensive retinopathy. In the cases associated with neovascularization, however, PRP or intravitreal antiVEGF injections or both should be undertaken to induce regression of the neovascularizations

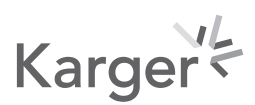


and potentially prevent further complications. We preferred bilateral PRP over anti-VEGF intravitreal injections in our case due to the socioeconomic status and the noncompliance of the patient. These patients should be followed closely as they could show recurrence of neovascularization that would require repeating the treatment.

This case, in addition to the few previous reports, provides evidence supporting the notion of retinal neovascularization secondary to severe systemic hypertension. The fact that malignant hypertension might cause such a condition with devastating sequelae should encourage ophthalmologists to follow similar cases closely and to assume a more active role in their management.

\section{Conclusion}

Malignant hypertension can rarely cause proliferative retinopathy with its devastating sequelae. Managing hypertensive retinopathy with antihypertensives alone, as is the current standard of care, might not be enough. Those patients with retinal neovascularizations need to be identified and treated with PRP and/or intravitreal anti-VEGF and followed closely. Early recognition and timely treatment are of paramount importance and require a close multidisciplinary management.

\section{Statement of Ethics}

This case report was conducted according to good clinical practice. The authors state that they have full control over all primary data and have no ethical conflicts to disclose. Written informed consent was obtained from the patient for publication of this case report and any accompanying images.

\section{Conflict of Interest Statement}

The authors have no conflicts of interest to declare.

\section{Funding Sources}

Open access funding provided by the Qatar National Library.

\section{Author Contributions}

The contributions of the authors were in line with the ICMJE authorship criteria. R.A.S. diagnosed the condition and performed the literature search, data collection and drafted the manuscript. O.M. provided ophthalmic care and critically reviewed and revised the manuscript. L.A. reviewed the patient from the rheumatology point of view and critically reviewed and revised the manuscript. All the authors contributed to data interpretation, and all the authors read and approved the final manuscript. 


\section{References}

1 Stryjewski TP, Papakostas TD, Vavvas D. Proliferative hypertensive retinopathy. JAMA Ophthalmol. 2016; 134(3):345-6.

2 Konstantinidis L, Guex-Crosier Y. Hypertension and the eye. Curr Opin Ophthalmol. 2016;27(6):514-21.

3 Georgiadis O, Kabanarou SA, Batsos G, Feretis E, Xirou T. Bilateral hypertensive retinopathy complicated with retinal neovascularization: panretinal photocoagulation or intravitreal anti-VEGF treatment? Case Rep Ophthalmol. 2014;5(2):231-8.

4 Lean JS, Gregor Z. The acute vitreous haemorrhage. Br J Ophthalmol. 1980;64(7):469-71.

5 Golshani C, Lieberman RM, Fischer RM, Brodie SE. Hypertensive crisis with massive retinal and choroidal infarction. Am J Ophthalmol Case Rep. 2017;6:58-60.

6 Avila SA, Golshani C, Friedman AH. Hypertensive crisis with massive retinal and choroidal infarction: a case update. Am J Ophthalmol Case Rep. 2019;13:22-4.

7 Abbassi S, Thinda S, Morse LS. Hypertensive retinopathy, choroidopathy, and optic neuropathy. JAMA Ophthalmol. 2015;133(10):e151494.

8 Jampol LM, Ebroon DA, Goldbaum MH. Peripheral proliferative retinopathies: an update on angiogenesis, etiologies and management. Surv Ophthalmol. 1994;38(6):519-40.

9 Brancato R, Menchini U, Bandello F. Proliferative retinopathy and toxemia of pregnancy. Ann Ophthalmol. 1987;19(5):182-3.

10 Luo BP, Brown GC. Update on the ocular manifestations of systemic arterial hypertension. Curr Opin Ophthalmol. 2004;15(3):203-10. 